\title{
Label-free optical monitoring of surface adhesion of extracellular vesicles by grating coupled interferometry
}

D. Patko ${ }^{1,2}$, B. Gyorgy ${ }^{3}$, A. Nemeth ${ }^{3}$, K. E. Szabó-Taylor ${ }^{3}$, A Kittel $^{4}$, E. I Buzas ${ }^{3 *}$, R. Horvath ${ }^{1 *}$

${ }^{1}$ Hungarian Academy of Sciences Research Centre for Natural Sciences Institute for Technical Physics and Materials Science, Hungary

${ }^{2}$ Doctoral School of Molecular-and Nanotechnologies, Pannon University, Veszprém, Hungary

${ }^{3}$ Semmelweis University, Department of Genetics, Cell- and Immunobiology, Budapest, Hungary

${ }^{4}$ Institute of Experimental Medicine, Hungarian Academy of Sciences, Budapest, Hungary

*These authors contributed equally and are co-authors for correspondence emails: edit.buzas@gmail.com; horvathr@mfa.kfki.hu 


\begin{abstract}
In this proof-of-principle study a label-free optical sensor is demonstrated to monitor the surface adhesion of extracellular vesicles secreted by live cells on to various extracellular matrix proteins.
\end{abstract}

\title{
1. Introduction
}

Sensitive monitoring of the presence and interactions of biological objects at the nanometer length scale is increasingly important for both basic research and industrial applications. Following refractive index variations caused by the biological object itself is an emerging strategy to create optical sensors where any further labelling of biomolecules or living cells is unnecessary. This approach may open the way for a natural, reliable and cost-effective investigation of nanosized structures. ${ }^{1}$

Most of the label-free optical sensors are exploiting a surface bound evanescent wave to follow the refractive index variations. ${ }^{2}$ The evanescent wave is localised in the close vicinity of the sensor surface, making it ideal for in situ monitoring of surface adhesion processes. A relatively wellknown evanescent wave sensor is the Surface Plasmon Resonance (SPR) using a gold film. However, planar optical waveguides offer higher sensitivities. ${ }^{3,4}$ The typical depth of penetration for a waveguide mode at optical frequencies is $80-300 \mathrm{~nm}$. In contrast to SPR, using waveguides the penetration depth can be easily tuned through waveguide design, matching in this way the sensing depth to the size of the adsorbed object. $5,6,7$

An emerging area where the label-free surface sensitive sensors could be exploited is the interaction of extracellular vesicles (EVs) with model surfaces, biomolecules, polymers and receptors. Extracellular vesicles are cell-derived membrane surrounded structures of various sizes. The two most extensively characterized types of EVs are the exosomes (50-100 nm vesicles generated by the exocytosis of multivesicular bodies) and microvesicles (MVs, plasma membrane-derived 100-1000 $\mathrm{nm}$ in diameter, often also referred to as shedding microvesicles, microparticles or ectosomes). The field of EVs represents one of the most rapidly emerging research areas in current biomedicine. These membrane bound structures are recently recognized conveyors of intercellular communication mediated either by membrane proteins or by their mRNA and microRNA cargo. ${ }^{8,9}$ The size range of EVs does not match that of the detection of instruments used in conventional cell biology and clinical diagnostic laboratories. Thus, there is a sudden demand and an intensive 
methodology development worldwide to meet these unmet needs. The evanescent field-based highly sensitive sensors may provide an exciting detection system for EVs.

We have recently introduced Grating Coupled Interferometry (GCI), a label-free evanescent-field based optical sensing concept. ${ }^{10-12}$ The sensing strategy combines cost-effectiveness, simplicity and reliability of grating-coupled planar optical waveguides with the high resolution of interferometric measurements. The heart of the GCI technology is a high quality planar optical waveguide shown in Fig. 1. In order to fabricate the sensor chip, 3 gratings are etched into a glass support, and the glass is coated with a $\mathrm{Ta}_{2} \mathrm{O}_{5}$ waveguide film of $155 \mathrm{~nm}$ thickness. Next, a relatively thick layer of $\mathrm{SiO}_{2}$ is used to coat the entire waveguide surface, except for a $5 \mathrm{~mm}$ long aperture (see Fig. 1). This aperture serves as a sensing window of the device where the evanescent field of the waveguide mode can interact with the surface-adsorbed analytes. The present waveguide design with TE polarization gives a penetration depth of $80 \mathrm{~nm}$ into the cover media. The bulk refractive index sensitivity is 0.072 , and the final refractive index resolution is approximately $10^{-7}$.

In this work we demonstrate the use of GCI in the field of EV research. The biological question we addressed was whether MVs, secreted by the human CCRF-CEM T cell line (ECACC), bind to components of the extracellular matrix. As yet, there is very limited data available on the adhesion of EVs to matrix proteins. ${ }^{13-15}$ Several types of EVs carry adhesion molecules on their surface (for a meta-analysis see ref. 16). Most interestingly, the presence of integrins and proteins involved in integrin-mediated signaling are characteristic for the surface of MVs. ${ }^{16}$ Therefore we hypothesised that EVs bind to extracellular matrix molecules (such as type I collagen or fibronectin), enabling them to participate in tissue regeneration and repair, cell migration, inflammation and blood clotting.

\section{Materials and methods}

The methodology developed in the present work to monitor the interaction of MVs with matrix proteins is summarized schematically in Fig. 2. One million CCRF-CEM cells / $\mathrm{mL}$ were cultured in serum-free RPMI for 24 hours. The serum-free culture condition did not induce a significant apoptosis as confirmed by the absence of annexin V-FITC binding using flow cytometry (data not shown). The $24 \mathrm{~h}$ conditioned medium was pelleted at $400 \mathrm{~g}$ for 15 minutes to remove cells, and the supernatant was filtered through a $800 \mathrm{~nm}$ pore-sized filter (Millipore, Billerica, CA) by gravity. The filtered supernatant was centrifuged at 20,500g for 60 minutes to pellet MVs, as described previously. ${ }^{17-21} \mathrm{MVs}$ were washed once in PBS filtered through a $0.1 \mu \mathrm{m}$ pore-size filter. Protein 
concentration of the MV preparations was measured by MicroBCA Proten Assay Kit (Pierce Biotechnology, Inc.). The average protein concentration in the samples used for GCI analysis was $15 \mu \mathrm{g} / \mathrm{mL}$. Samples were stored at $-80^{\circ} \mathrm{C}$ until use, but for not longer than 2 weeks. Finally, the GCI sensor surface, coated with the biological molecule of interest, was exposed to the MVcontaining samples.

To investigate the quality of the vesicle solution, shortly after centrifugation the supernatants were carefully removed, and the pellets were fixed at RT for 60 minutes with $4 \%$ PFA in $0.01 \mathrm{M}$ PBS. After washing with PBS, the preparations were post-fixed in $1 \% \mathrm{OsO}_{4}$ (Taab, Aldermarston, UK) for 30 minutes. Following rinsing with distilled water, the pellets were dehydrated in graded ethanol, including block-staining with $1 \%$ uranyl-acetate in $50 \%$ ethanol for 30 minutes, and embedded in Taab 812 (Taab). After overnight polymerization at $60{ }^{\circ} \mathrm{C}$ and sectioning for EM, the ultrathin sections were analyzed with a HITACHI 7100 electron microscope equipped by Megaview II (lower resolution, Soft Imaging System) digital camera. Fig. 3a demonstrates the excellent quality of the MV preparation. The size distribution of MVs was further analyzed using a scanning ion occlusion sensing approach (qNano, Izon Science Ltd., Christchurch, New Zealand). ${ }^{22}$

Samples were counted for 5 minutes using 7.15 mbar pressure. Voltage was set in between 0.1-0.25 $\mathrm{V}$ in order to achieve a stable $100 \mathrm{nA}$ current. Particle size histograms were recorded when RMS noise was below $12 \mathrm{pA}$, and particle rate in time was linear. Using the 200 and $400 \mathrm{~nm}$ pore membranes successively, most of the MVs were around 200-300 nm, but a smaller fraction of vesicles with larger diameters was also present (see Fig. 3b). This size range is in accordance with EM images in this study and previous findings. ${ }^{17,23}$

\section{Results and discussions}

In order to monitor MV adsorption on to the GCI sensor, the cleaned sensor chip was first placed into an optical arrangement shown schematically in Fig. 1. Here, two beams of a linearly polarized He-Ne laser illuminate the $1^{\text {st }}$ and $2^{\text {nd }}$ gratings, which couple the waves into the planar optical waveguide. The second beam is phase modulated using a Liquid Crystal Modulator (LCM), creating a time-dependent interference signal after the $2^{\text {nd }}$ grating. This signal is outcoupled at the $3^{\text {rd }}$ grating, and picked up by a fiber-coupled optical detector. The interference signal is analyzed by a personal computer, which calculates the phase shift the waveguide mode experienced at the sensing window of the chip. The temperature of the chip was stabilized with a Peltier-element at $25^{\circ} \mathrm{C}$. Over the sensing window a PEEK microcuvette with a Kalretz O-ring is mounted. The inlet 
of this cuvette is equipped with a septum injection port in order to introduce minute amount of samples into the cuvette (see Fig. 1). The continuous washing of the sensor with pure buffer can be also done through the septum by using needle ended tubing (see Fig. 1). It is important to note that the septum was fixed by a special holder in order to minimize the mechanical disturbance of the interferometer during sample introduction.

First, pure PBS was injected into the cuvette through the septum using a peristaltic pump and a fluidic tubing that ends up in a needle (see Fig. 1). After recording a stable baseline, the solutions of type I collagen $(0.3 \mathrm{mg} / \mathrm{ml})$ and fibronectin $(40 \mu \mathrm{g} / \mathrm{ml})$ (both from Sigma-Aldrich) were injected using a Hamilton syringe. The detected phase continuously increased (see Fig. 4a) indicating that the proteins were adsorbing on to the surface. After approx. 15 minutes, pure buffer was pumped into the cuvette again to wash off any irreversibly adsorbed proteins. Then, the MV containing sample was injected. MVs, adsorbing in the sensing window, increased the refractive index inside the evanescent field, thus, increasing the phase. After the MV exposure, again, pure buffer was flowed over the cuvette to remove any irreversibly adsorbed vesicles and the phase shift was recorded again.

Fig. 4 shows the recorded phase shifts. As seen in the figure, the vesicles did not adsorb irreversibly on to the type I collagen coating. In contrast, an approximately 3 radians phase shift was observed with the fibronectin-coated sensor chip. Vesicle adsorption was also tested on bare and positively charged poly-L-lysine (PLL) coated waveguide surfaces. No irreversible adsorption was seen on the bare $\mathrm{Ta}_{2} \mathrm{O}_{5}$ chip, but the vesicles irreversibly bound to PLL causing a phase shift of 2.8 radians (see Fig. 4). This binding may be explained by the known externalization of the negatively charged phosphatidylserine by MVs. ${ }^{17,18,23}$ Our data suggest that PLL may be used to immobilize MVs, and may serve as an inexpensive alternative to annexin V. Importantly, we also showed that MVs bind to fibronectin. This is in line with the reported presence of integrins on the surface of leukocytederived MVs that may possibly mediate the interaction with fibronectin. The observed failure of MVs to adsorb on to type I collagen coating might be related to the conformation of collagen on the surface of $\mathrm{Ta}_{2} \mathrm{O}_{5}$. It is important to stress that using the present optical arrangement $2 \times 10^{-4}$ refractive index shift in the evanescent field corresponds to one radian phase shift in the recorder GCI signal. Therefore, considering the above phase shifts caused by the adsorbed MVs, one can conclude that high refractive index sensitivity is essential to resolve the signal of surface adsorbed MVs. 


\section{Conclusions}

In summary, in this proof-of-principle study a novel method was introduced to study EVs, and was used for monitoring of the interaction of cell-derived MVs with extracellular matrix molecules. The applied GCI technique is completely label-free; the vesicles were investigated in their native state. It is important to stress that EVs represent novel diagnostic biomarkers of various diseases. They are highly abundant in various biological fluids including blood plasma, urine, cerebrospinal fluid, synovial fluid, saliva, breast milk, etc. Their levels, protein compositions and miRNA signatures were shown to associate with numerous pathologies including tumors, autoimmune disorders, cardiovascular diseases, infections. ${ }^{16}$ However, their routine clinical analysis is limited, and thus, there is an urgent need for the development of new techniques for the analysis of EVs. In this study we investigated MV-extracellular matrix protein interaction in order to prove the applicability of GCI for EV analysis. Further applications of this novel technique include studying EV surface molecules by investigating the binding of EVs to antibody-coated surfaces as well as investigation of receptor-ligand interactions in the field of EVs. The GCI technique can be especially advantageous for the above applications since it not only offers superior refractive index sensitivity with excellent time resolution; but contains no moving parts, built up from simple and inexpensive components. After further developments the GCI technique could be made suitable for handheld devices as well as for high throughput screening instruments, facilitating industrial and diagnostic applications as well.

\section{Acknowledgements}

This research was realized in the frames of TÁMOP 4.2.4. A/1-11-1-2012-0001 „National Excellence Program - Elaborating and operating an inland student and researcher personal support system." The project was subsidized by the European Union and co-financed by the European Social Fund. This work was also supported by the Hungarian Scientific Research Funds OTKA K73247, NK 84043, Kerpel-Fronius Ödön and Jedlik Ányos Fellowship Programs, Baross Gábor (REG-KM-09-1-2009-0010) and FP7-PEOPLE-2011-ITN - PITN-GA-2011-289033 "DYNANO”, the National Development Agency grants TÁMOP-4.2.2/B-10/1-2010-0025 and REG_KD_09-22009-0022-NANOFLAG, and by the Lendület program of the Hungarian Academy of Sciences. The support of Creoptix GmbH is also gratefully acknowledged. 


\section{References}

1. H. K. Hunt and A. M. Armani, Nanoscale, 2010, 2, 1544-1559.

2. M. C. Estevez, M. Alvarez and L. M. Lechuga, Laser \& Photonics Reviews, 2012, 6, 463-487.

3. W. Lukosz, Biosens. and Bioelectr., 1991, 6, 215-225.

4. W. Huber, R. Barner, Ch. Fattinger, J. Hübscher, H. Koller, F. Müller, D. Schalatter and W. Lukosz, Sens. Actuators B., 1992, 6, 122-126.

5. R. Horvath, H. C. Pedersen, and N. B. Larsen, Appl. Phys. Lett, 2002, 81, 2166-2168.

6. R. Horvath, H. C. Pedersen, N. Skivesen, C. Svanberg, and N. B. Larsen, J. Micromech. Microeng., 2005, 15, 1260-1264.

7. R. Horvath, H. C. Pedersen, N. Skivesen, D. Selmeczi, and N. B. Larsen, Appl. Phys. Lett., 2005, 86, 071101.

8. C. Théry, M. Ostrowski, E. Segura, Nat. Rev. Immunol., 2009, 9,581-93.

9. H. Valadi, K. Ekström, A. Bossios, M . Sjöstrand, J. J. Lee, J. O. Lötvall, Nat. Cell Biol. 2007, 9, 654-9.

10. P. Kozma, A. Hamori, K. Cottier, S. Kurunczi, and R. Horvath, Appl. Phys. B., 2009, 97, 5-8.

11 P. Kozma, A. Hamori, S. Kurunczi, K. Cottier, and R. Horvath, Sens. Actuators B., 2011, 155, $446-450$.

12. D. Patko, K. Cottier, A. Hamori, and R. Horvath, Optics Express, 2012, 20, 23162-23173.

13 A. Clayton, A. Turkes, S. Dewitt, R. Steadman, M. D. Mason, M. B. Hallett, Faseb J., 2004, 18, 977-979.

14. R. B. Koumangoye, A. M. Sakwe, J. S. Goodwin, T. Patel ,J. Ochieng. PLoS One., 2011, 6, 24234.

15. A. Janowska-Wieczorek, M. Wysoczynski, J. Kijowski, L. Marquez-Curtis, B. Machalinski, J. Ratajczak, M. Z. Ratajczak, Int. J. Cancer, 2005, 113, 752-760.

16. B. Gyorgy, T. G. Szabo, M. Pasztoi, Z. Pal, P. Misjak, B. Aradi, V. Laszlo, E. Pallinger, E. Pap, A Kittel, G. Nagy, A. Flus, E. Buzas, Cell. Mol. Life Sci., 2011, 68. 2667-2688.

17. B. Gyorgy, K. Modos, E. Pallinger, K. Paloczi, M. Pasztoi et al., Blood., 2011 , 117, e39-e48.

18. A. S. Leroyer, P. E. Rautou, J. S. Silvestre, Y. Castier, G. Leseche et al., J. Am. Coll. Cardiol., 2008, 52, 1302-1311.

19. Y. Yuana, T. H. Oosterkamp, S. Bahatyrova, B. Ashcroft, P. Garcia Rodriguez et al., J. Thromb Haemost., 2010, 8, 315-23.

20. M. E. Tesselaar, F. P. Romijn,I. K. van der Linden, F. A. Prins, R. M. Bertina et al., J. Thromb Haemost., 2007, 5,520-527. 
21. P. M. van der Zee, E. Biró, L. A. Trouw, Y. Ko Y,R. J. de Winter, et al., Clin. Immunol., 2010, 135, 490-495.

22. J. de Vrij, S. L. Maas, M. van Nispen,M. Sena-Esteves, R. W. Limpens, A. J. Koster, S. Leenstra, M. L. Lamfers and M. L. Broekman, Nanomedicine (Lond). 2013 Feb 5.

23. E. van der Pol, A. N. Boing, P. Harrison, A. Sturk and R. Nieuwland, Pharmacol. Rev., 2012, 64, 676-705. 


\section{Figure captions}

Figure 1. Schematic cross-sectional illustration of the GCI sensor arrangement. The cuvette positioned above the optical waveguide sensor is equipped with a septum injection port for sample introduction using a Hamilton syringe. The washing off the irreversibly bound vesicles from the sensor by pure buffer is also done through the septum using a peristaltic pump equipped with needle ended tubing. The top view photograph of the GCI sensor chip is also shown. The two incoupling and the single outcoupling grating, as well as the $5 \mathrm{~mm}$ long sensing window, are clearly visible.

Figure 2. Steps of isolation of MVs that were analyzed for their adhesion to the protein pre-coated GCI sensor surface. The surface adsorbed microvesicles are detected by the evanescent field of the propagating waveguide mode.

Figure 3.(a) Transmission electron microphotograph of CCRF-CEM human cell line derived MVs (b) Size distribution of the MVs determined by scanning ion occlusion sensing approach. The size distribution curves were show combined data obtained by using the two single nanopore (200 and $400 \mathrm{~nm})$ membranes.

Figure 4. (a) In-situ phase change during the coating process of the GCI sensor with collagen and fibronectin, followed by the introduction of the MV solution and subsequent washing with pure buffer. The coating with Poly-L-lysine (PLL) was not done in-situ, the waveguide was already coated when inserted into the GCI setup. The in situ phase signals, when the bare and the PLL coated waveguide was exposed to the MV solution, are also shown. The numbered arrows indicate: 1) introduction of coating molecules, 2) washing off the irreversibly bound coating molecules using pure buffer, 3) injection of MV suspension, 4) washing off the irreversibly bound MVs using pure buffer (b) The recorded phase differences when the bare and the matrix protein or Poly-L-lysine (PLL)-coated GCI chip was exposed to the MV suspension (grey columns); the phase signals after washing off the MV suspension by pure buffer (black columns). 


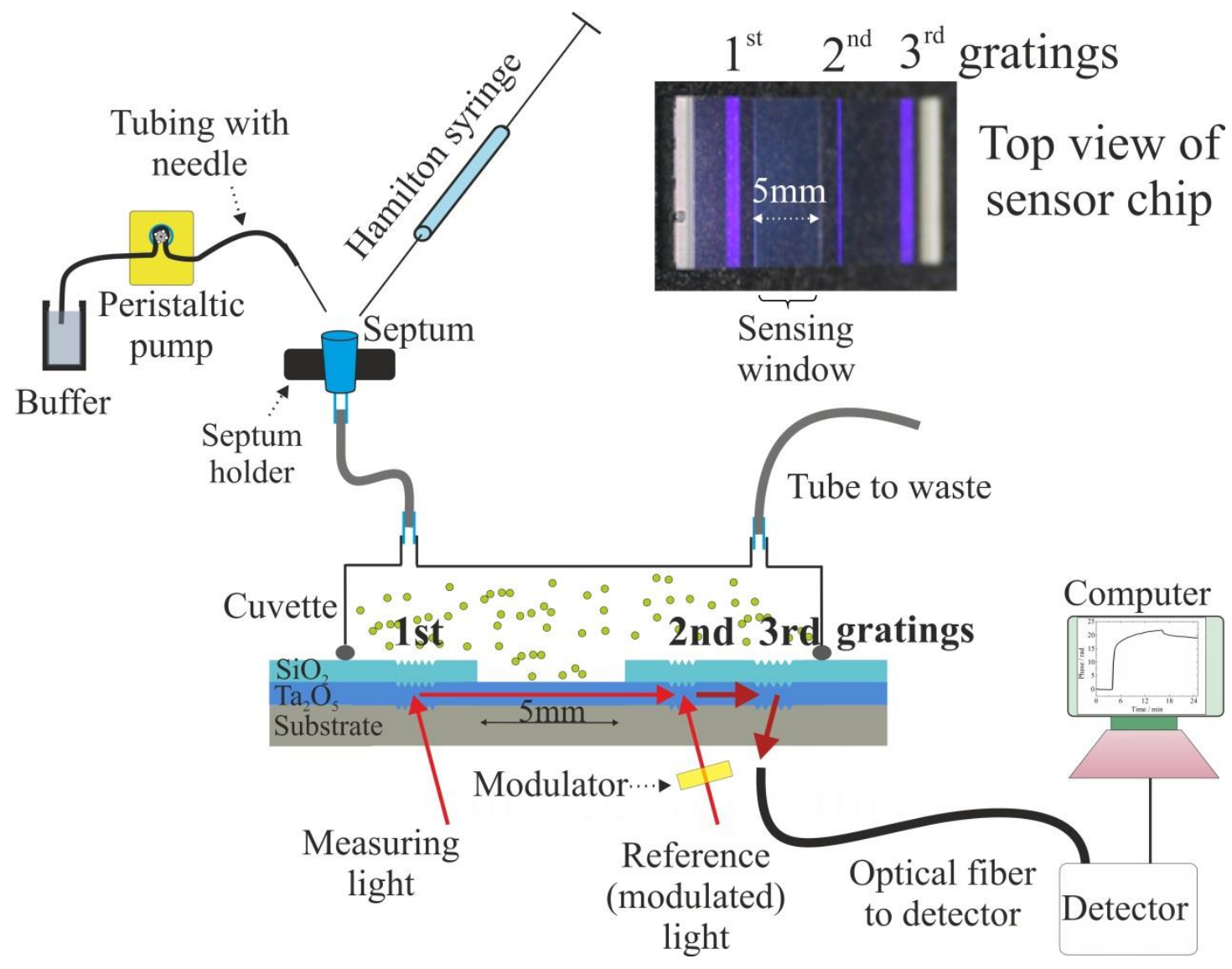

Figure 1. 

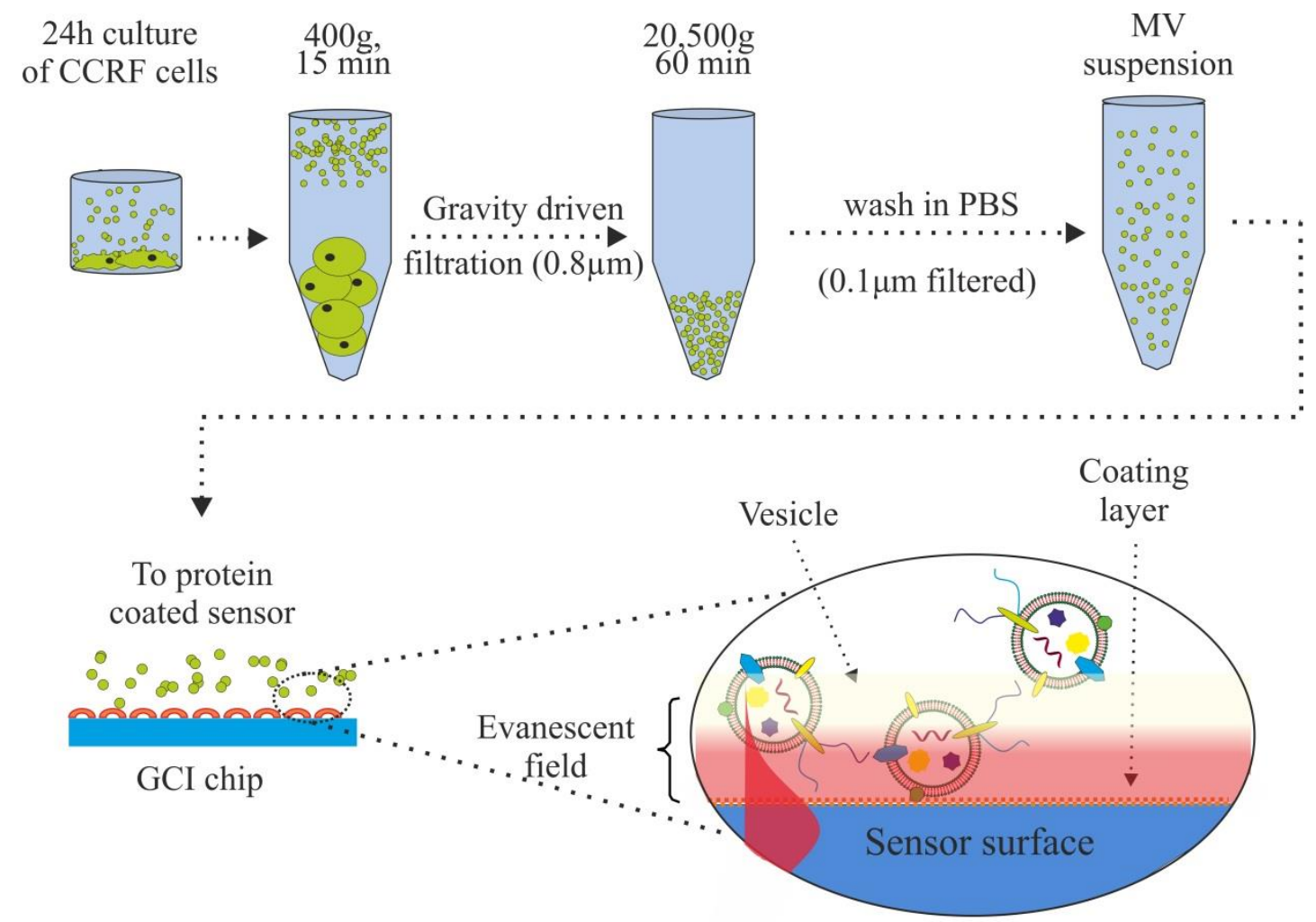

Figure 2. 

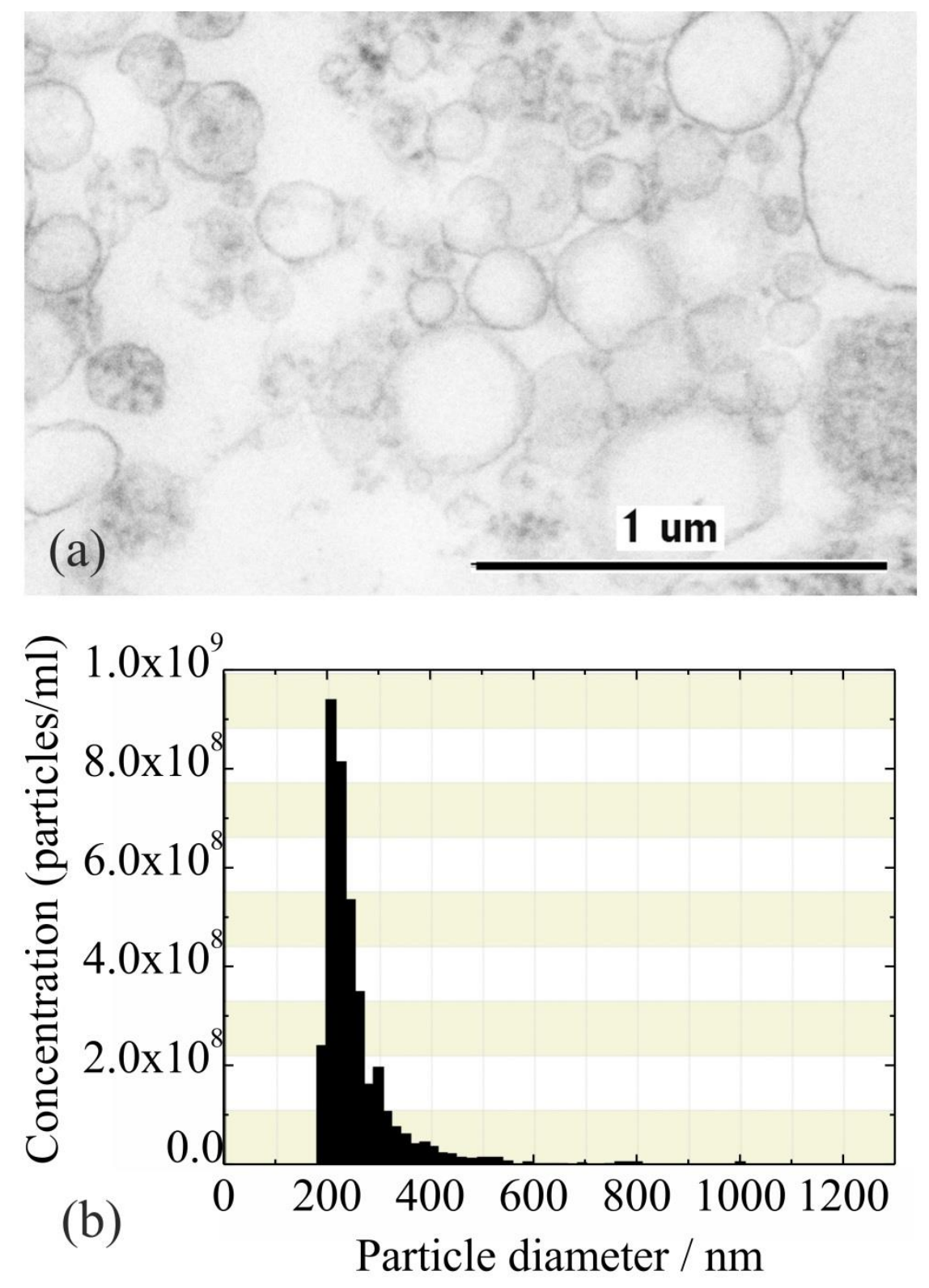

Figure 3. 

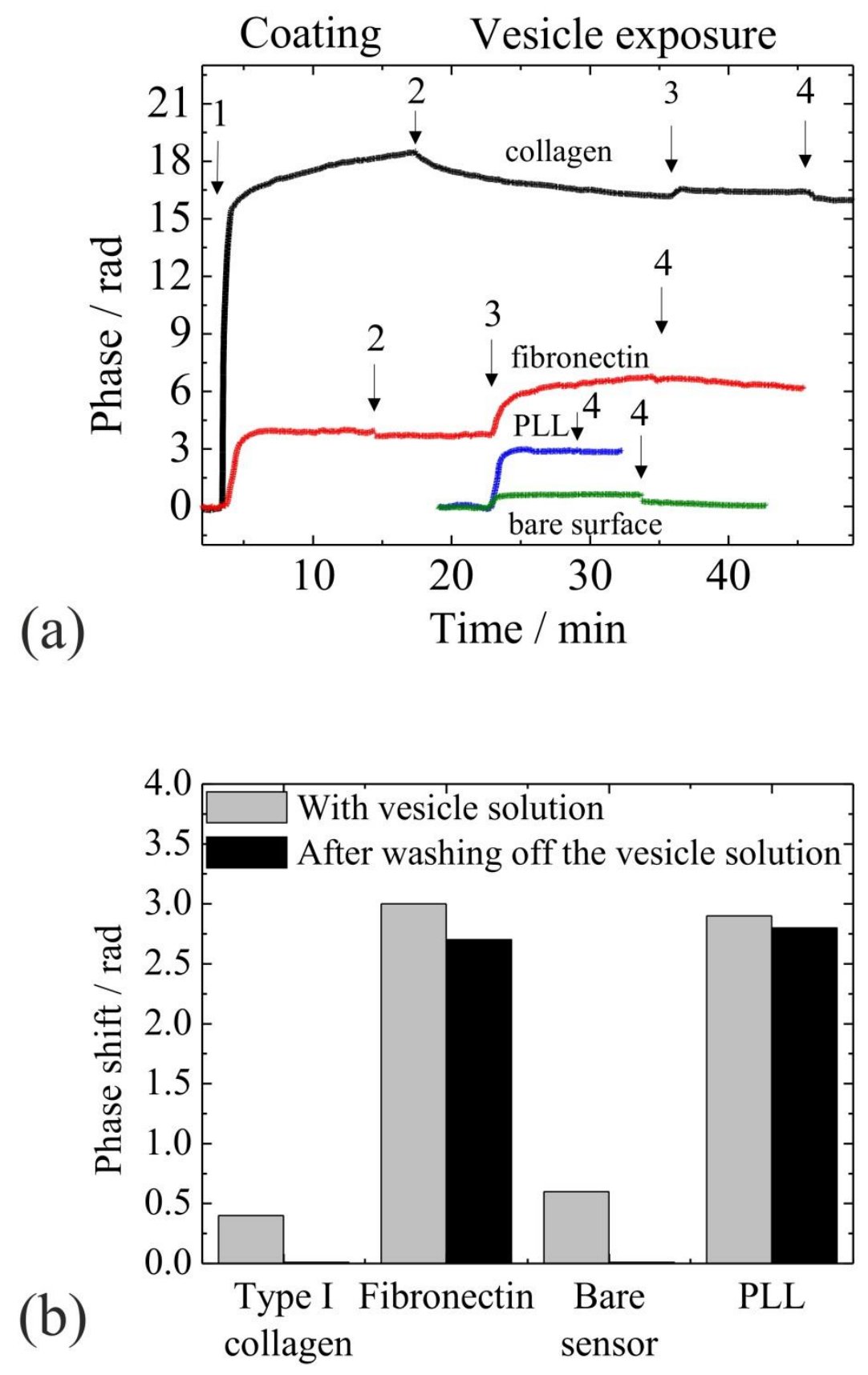

Figure 4. 